\title{
Serum complement activation on heterologous platelets is associated with arterial thrombosis in patients with systemic lupus erythematosus and antiphospholipid antibodies
}

\author{
EIB Peerschke ${ }^{1,2}$, W Yin $^{1,3}$, DR Alpert ${ }^{4,5}$, RAS Roubey $^{6}$, JE Salmon ${ }^{4}$, and B Ghebrehiwet ${ }^{7}$ \\ ${ }^{1}$ Department of Pathology and Laboratory Medicine, Weill Medical College of Cornell University, New York, \\ New York, USA \\ ${ }^{2}$ Department of Pathology, The Mount Sinai School of Medicine, New York, New York, USA \\ ${ }^{3}$ Department of Mechanical and Aerospace Engineering, Oklahoma State University, Stillwater, Oklahoma, \\ USA \\ ${ }^{4}$ The Hospital for Special Surgery, Weill Medical College of Cornell University, New York, New York, USA \\ ${ }^{5}$ Jersey Shore University Medical Center, Neptune, New Jersey, USA \\ ${ }^{6}$ Department of Medicine and Thurston Arthritis Research Center, University of North Carolina at Chapel \\ Hill, Chapel Hill, North Carolina, USA \\ ${ }^{7}$ Departments of Medicine and Pathology, Stony Brook University, Stony Brook, New York, New York, USA
}

\section{Abstract}

Complement plays a major role in inflammation and thrombosis associated with systemic lupus erythematosus (SLE) and the antiphospholipid syndrome (APS). A cross-sectional retrospective analysis was performed to evaluate serum complement fixation on platelets and thrombotic incidence using banked sera and clinical data from patients with SLE ( $n=91)$, SLE with antiphospholipid antibodies (aPL) or APS $(n=78)$ and primary aPL $(n=57)$ or APS $(n=96)$. In-situ complement fixation was measured as $\mathrm{C} 1 \mathrm{q}$ and $\mathrm{C} 4 \mathrm{~d}$ deposition on heterologous platelets using an enzyme-linked immunosorbent assay approach. Platelet activation by patient serum in the fluid phase was assessed via serotonin release assay. Enhanced in-situ complement fixation was associated with the presence of $\operatorname{IgG~aPL}$ and IgG anti- $\beta 2$ glycoprotein 1 antibodies $(P<0.05)$ and increased platelet activation $(P<0.005)$. Moreover, enhanced complement fixation, especially $\mathrm{C} 4 \mathrm{~d}$ deposition on heterologous platelets, was positively associated with arterial thrombotic events in patients with SLE and aPL $(P=0.039)$. Sera from patients with aPL possess an enhanced capacity for in-situ complement fixation on platelets. This capacity may influence arterial thrombosis risk in patients with SLE.

\section{Keywords}

antiphospholipid syndrome; systemic lupus erythematosus; thrombosis 


\section{Introduction}

Complement plays a major role in inflammation and thrombotic complications associated with systemic lupus erythematosus (SLE) and antiphospholipid syndrome (APS). APS is an autoimmune thrombophilic condition associated with the presence of antiphospholipid antibodies (aPL), but not all patients with aPL have or develop APS. ${ }^{1,2}$ The risk of thrombosis is particularly high in patients with SLE and aPL. ${ }^{3}$ Understanding the pathophysiology of thrombosis in this setting and developing targeted preventive strategies is thus highly relevant.

In-vivo and in-vitro studies show that aPL activate endothelial cells and platelets and contribute to thrombosis and tissue injury. ${ }^{4-9}$ A major antigen recognized by aPL is $\beta 2$ glycoprotein 1 ( $\left.\beta_{2} \mathrm{GP} 1\right)$, a member of the complement control protein superfamily. ${ }^{1}$ Proposed mechanisms for the thrombotic potential of anti- $\beta_{2} \mathrm{GP} 1$ antibodies include interference with endogenous anticoagulation mechanisms by anti- $\beta_{2} \mathrm{GP} 1$ immune complexes, the triggering of signalling events on cells including endothelial cells, platelets, trophoblasts and leukocytes and complement activation with an ensuant inflammatory reaction on the vascular and trophoblast surface. ${ }^{1,10-12}$

In addition, complement activation has been implicated in thrombotic complications associated with APS. ${ }^{1,10}$ In-vivo complement activation is required for aPL-induced foetal loss and growth retardation. ${ }^{11}$ Moreover, an increase in complement activation products in the serum of patients with aPL has been associated with the development of stroke and transient ischemic events. ${ }^{13}$ A high frequency of complement fixing aPL has been described in patients with APS. ${ }^{14}$ Recently, platelet-associated C4d was reported in $18 \%$ of patients with SLE and was significantly associated with the presence of a lupus anticoagulant and anticardiolipin antibodies. 15

Indeed, platelets have been described as targets of circulating aPL. ${ }^{4}$ The interaction of aPL with platelets has been reported to occur by several mechanisms, including direct recognition of platelet antigens, cross-linking apolipoprotein E R2 ${ }^{12}$ and antibody interaction with platelet Fc $\gamma$ RII receptors. ${ }^{4}$ Depending on antibody isotype, platelet-associated aPL may thus play a role in the activation of the classical complement pathway, leading to deposition of classical complement components, including $\mathrm{C} 4 \mathrm{~d}$, a stable degradation product of $\mathrm{C} 4 \mathrm{~b}$, on the platelet surface.

The present study was conducted to test the hypothesis that enhanced in-situ classical pathway complement activation on platelets is associated with thrombotic events in patients with SLE and/or aPL antibodies. A recently developed in-vitro assay measuring complement fixation on immobilized heterologous platelets ${ }^{16}$ was used to evaluate in-situ complement activation by sera from patients with SLE, SLE with aPL or APS and patients with primary aPL or APS. The data show an enhanced capacity of sera from patients with aPL to activate complement on heterologous platelets and suggest a positive correlation with arterial thromboses in patients with SLE.

\section{Methods}

\section{Study population}

A cross-sectional retrospective analysis was performed using banked sera from patients having SLE, with or without aPL or APS, and patients with primary aPL or APS. The study was performed in accordance with the guidelines of the Weill Medical College of Cornell University and Hospital for Special Surgery Institutional Review Boards for the Protection of Human Subjects and the Helsinki Declaration of 1975, as revised in 1983. 
Patients with SLE met American College of Rheumatology criteria for SLE. ${ }^{17}$ All patients with APS had a history of IgG or IgM anticardiolipin antibodies (aCL), anti- $\beta 2 \mathrm{GP} 1 \mathrm{IgG}$ or IgM antibodies and/or positive lupus anticoagulant tests documented on two or more occasions at least 6 weeks apart and met Sapporo clinical criteria. ${ }^{2}$ All patients having aPL without APS met the above laboratory criteria but not Sapporo clinical criteria. SLE patients without aPL had at least one documented negative test result for a lupus anticoagulant and negative IgG, $\operatorname{IgM}$ and $\operatorname{Ig} \mathrm{A}$ aCL antibodies.

Banked sera and clinical data for patients having SLE with aPL or APS $(n=78)$ and SLE patients without aPL $(n=91)$ were obtained from the Hospital for Special Surgery Autoimmune Disease Registry. The clinical and laboratory characteristics of these patient populations were recently described in detail. ${ }^{18}$

Sera and corresponding clinical and laboratory data for patients with primary aPL or APS ( $n$ = 153) were obtained from the national Antiphospholipid Syndrome Collaborative Registry (APSCORE).

\section{Immunoassays}

aPL levels for both SLE patients and patients in the APSCORE registry were determined on the same serum samples used for complement activation. For the SLE patient cohort, IgG and IgM APhL levels were determined by a commercial immunoassay kit (Louisville APhL Diagnostics, Seabrook, Texas, USA) using a proprietary phospholipid mixture and $\beta 2 \mathrm{GP} 1$ as substrate coated on polystyrene platelets. APhL levels have been shown to correlate with aCL levels and may be more specific for APS. ${ }^{19,20}$ Results are expressed as standard GPL and MPL units. ${ }^{21}$ IgG and IgM anti- $\beta 2$ GP1 levels were assayed using a commercial immunoassay kit (Inova Diagnostics, San Diego, California, USA) with levels reported as standard IgG (SGU) or IgM (SMU) units.

For aPL and APS patients enrolled in the APSCORE registry, aCL and $\beta 2 \mathrm{GP} 1$ antibody levels were determined centrally by the registry. aCL levels were determined using a kit from Louisville aPL Diagnostics. $\beta 2 \mathrm{GP} 1$ antibody levels were measured with an immunoassay kit from Inova Diagnostics.

Positive levels for all aPL antibodies (APhL, aCL and anti- $\beta 2 \mathrm{GP} 1 \mathrm{IgG}$ and $\operatorname{IgM}$ ) were defined as $>20$ units, following normalization to appropriate assay standards supplied by the respective manufacturers.

\section{Complement activation on heterologous platelets}

A solid phase assay was used to measure the capacity of patient sera to activate the classical complement pathway on immobilized heterologous platelets. Platelets were obtained from healthy volunteers as previously described. ${ }^{16}$ Briefly, blood was collected into $3.2 \%$ sodium citrate (9:1 blood: anticoagulant ratio), after obtaining informed consent. Platelet rich plasma was prepared by centrifugation at $280 \mathrm{~g}$ for $8 \mathrm{~min}$. Platelets were washed by centrifugation (1000 g, $15 \mathrm{~min})$ in the presence of $10 \mathrm{mM}$ ethylenediaminetetra acetic acid (EDTA). The resulting cell pellet was resuspended in 0.01 M HEPES (N-2 hydroxyethyl piperazine ethane sulfonic acid)-buffered modified Tyrode's solution (HBMT) containing $1 \mathrm{mM} \mathrm{MgCl} 2,1 \mathrm{mM}$ $\mathrm{CaCl}_{2}$ and $2 \mathrm{mg} / \mathrm{mL}$ bovine serum albumin (BSA) (fatty acid free; Sigma-Aldrich, St Louis, Missouri, USA). No attempt was made to fully prevent platelet activation during the washing procedure. Washed platelets were immobilized on microtitre plate wells using poly-L lysine, as described. ${ }^{16}$ Adherent platelets were fixed with $0.5 \%$ glutaraldehyde in $0.01 \mathrm{M}$ phosphate buffered $0.14 \mathrm{M} \mathrm{NaCl}$ (PBS), pH 7.5. Microtitre wells were blocked with glycine $1 \%$ BSA and washed with HBMT. ${ }^{22}$ Immobilized platelets were exposed $\left(60 \mathrm{~min}, 37^{\circ} \mathrm{C}\right)$ to patient sera or 
sera from healthy volunteers $(n=50)$. All serum samples were diluted $(1 / 10)$ in HBMT containing $1 \mathrm{mM} \mathrm{CaCl}_{2}, 1 \mathrm{mM} \mathrm{MgCl}$ and $50 \mu \mathrm{M}$ D-phenylalanyl-L-prolyl-L arginine chloromethyl ketone (PPACK) (Calbiochem, San Diego, California, USA) to prevent thrombin-mediated complement activation. ${ }^{23}$ Complement fixation was assessed by examining the deposition of classical pathway complement components $\mathrm{C} 1 \mathrm{q}$ and $\mathrm{C} 4 \mathrm{~d}$ using commercially available monoclonal antibodies (Qui-del Corp., Santa Clara, California, USA; final concentration of $10 \mu \mathrm{g} / \mathrm{mL}$ ). Primary antibody binding was detected with an alkaline phosphatase-conjugated goat antimouse secondary antibody $\mathrm{F}\left(\mathrm{ab}{ }^{\prime}\right)_{2}$ fragment and paranitrophenyl phosphate (p-NPP) substrate, both from Sigma-Aldrich. Substrate conversion was measured spectrophotometrically at $405 \mathrm{~nm}$. Nonimmune mouse $\operatorname{IgG}_{1} \kappa(M O P C 21$, SigmaAldrich; $10 \mu \mathrm{g} / \mathrm{mL}$ ) served as a negative control antibody. Assay reactivity noted in the presence of nonimmune mouse $\mathrm{IgG}$ was subtracted from all experimental results. Immobilized platelets were screened for intrinsic C1q or C4d reactivity by incubation with HBMT instead of serum and no reactivity was detected.

Assay results were normalized to an internal assay standard to facilitate interassay comparisons. This standard was prepared by pooling serum from 25 healthy donors and was stored in aliquots at $-80^{\circ} \mathrm{C}$.

Reference ranges for the assay were established using serum samples from 50 healthy volunteers, who had not taken any medication for at least 10 days. Volunteers ranged in age from 25 to 67 years. Equal numbers of male and female volunteers were studied.

\section{Platelet activation}

To evaluate platelet activation in the fluid phase, a serotonin release assay (SRA) was performed as previously described ${ }^{24}$ Briefly, ${ }^{14} \mathrm{C}$-serotonin $(33 \mathrm{nCi} / \mathrm{mL}$ ) (Amersham Bioscience, Piscataway, New Jersy, USA) was added to platelet-rich plasma, prepared by centrifugation of anticoagulated ( $3.2 \%$ sodium citrate) whole blood at $280 \mathrm{~g}$ for $8 \mathrm{~min}$. After incubation at $37^{\circ} \mathrm{C}$ for $30 \mathrm{~min}$, samples were centrifuged in the presence of $10 \mathrm{mM}$ EDTA (1000 g, $20 \mathrm{~min}$ ), and platelets were resuspended in HBMT to a count between 200 and 300 $\mathrm{K} / \mu \mathrm{L} .{ }^{14} \mathrm{C}$-serotonin-labeled platelets were subsequently exposed to healthy donor or patient sera, diluted 1:10 in HBMT, to mimic conditions used in complement fixation studies. Following incubation at $37{ }^{\circ} \mathrm{C}$ for $60 \mathrm{~min}$, samples were fixed with ice-cold $1 \%$ formalin (Sigma-Aldrich) and centrifuged (12,000 g, $5 \mathrm{~min}$ ). The platelet-free supernatant was evaluated for released ${ }^{14} \mathrm{C}$-serotonin using liquid scintillation counting.

\section{Statistical evaluations}

Continuous data were evaluated using the unpaired student's $t$-test for parametric data and the Mann-Whitney $U$-test for nonparametric data. Nominal data were evaluated by chi-squared or Fisher's exact test. All analyses were two-tailed and $P<0.05$ were considered significant.

\section{Results \\ Patient demographics}

A total of 169 serum samples from patients with SLE were analysed. Patient demographics relevant to the present study are summarized in Table 1 . Ninety-one patients were aPL negative and 78 were aPL positive. aPL refers collectively to $\mathrm{aPhL}, \mathrm{aCL}$ and $\beta 2 \mathrm{GP} 1$ antibodies. $\mathrm{APhL}$ IgG and IgM levels were positive, respectively, in 87 and 64\% of SLE patients with aPL/APS. Levels of anti- $\beta 2 \mathrm{GP} 1 \mathrm{IgG}$ and IgM were positive in 53 and $46 \%$ of these patients, respectively. The presence or absence of a lupus anticoagulant was not be assessed at the time of study, as only serum samples, and not plasma samples, were available for analyses. Of the SLE patients whose sera were aPL positive, 27 patients had aPL without APS and 51 had APS by Sapporo 
criteria. $^{2}$ In the latter group, 41 patients experienced at least one thrombosis (32 arterial and 17 venous) and 23 patients experienced at least one pregnancy morbidity. aPL IgG levels trended towards higher levels in patients with SLE and APS $(P=0.057)$.

In addition, a total of 153 banked sera from patients with primary aPL (96 without APS, 57 with APS) were analysed. Relevant patient demographics are summarized in Table 2 . At the time of study, 88 and $45 \%$ of patients with APS exhibited positive serum titres for cardiolipin IgG and IgM, respectively. In addition, 73 and $56 \%$ of these patients were positive for serum $\operatorname{IgG}$ and $\operatorname{IgM}$ antibodies against $\beta 2 \mathrm{GP} 1$. Similarly, serum levels for anticardiolipin IgG and IgM were positive in 51 and $37 \%$ of patients with aPL without APS, respectively. Serum levels for $\beta 2 \mathrm{GP} 1 \mathrm{IgG}$ and IgM were positive in 33 and $35 \%$ of these patients, respectively. Serum levels for anticardiolipin and anti-ß2GP1 IgG were higher in aPL patients with APS than in aPL patients without APS, whereas the incidence of lupus anticoagulant positivity was similar. Of the 96 patients with aPL and APS, 74 experienced at least one thrombosis (44 arterial and 36 venous) and 37 patients experienced at least one pregnancy morbidity.

\section{Complement fixation}

Results for in-situ complement fixation by sera from patients with SLE and healthy controls are shown in Figure 1. Deposition of classical pathway complement components, C1q (panel A) and C4d (panel B), is expressed as a ratio relative to an internal assay standard. Mean and median values for $\mathrm{C} 1 \mathrm{q}$ and $\mathrm{C} 4 \mathrm{~d}$ deposition are illustrated in Figure 2. As described previously, ${ }^{16} \mathrm{C} 1 \mathrm{q}$ and $\mathrm{C} 4 \mathrm{~d}$ deposition occurred on immobilized platelets following exposure to normal human serum. The extent of complement deposition was previously found to be similar using autologous and heterologous serum from untransfused donors, suggesting a lack of effect from or sensitivity to $\mathrm{ABO}$ or HLA mismatch. ${ }^{16}$ In addition, previous studies showed similar levels of complement activation measured on platelets exposed to serum or plasma. ${ }^{16}$

In the present study, the reference interval for normalized $\mathrm{C} 1 \mathrm{q}$ and $\mathrm{C} 4 \mathrm{~d}$ deposition (ratio) on heterologous platelets determined using sera from healthy volunteers $(n=50)$ was $1.0 \pm 0.30$ and $1.13 \pm 0.60$, respectively. A small but significant increase in C1q deposition was noted using sera from patients with SLE without aPL $(1.21 \pm 0.47 ; P=0.006)$. However, a marked decline in $\mathrm{C} 4 \mathrm{~d}$ deposition was seen in this population $(0.61 \pm 0.32 ; P<0.0001)$ likely due to overall decreased serum complement levels (Table 1) relative to published reference ranges.

25 Decreased serum complement is often seen in the setting of SLE and related to consumption and/or genetics. ${ }^{26,27}$ In contrast, aPL positive sera from patients with SLE showed significantly increased $\mathrm{C} 1 \mathrm{q}$ deposition $(1.85 \pm 0.81)$ (Figure 1A) compared with sera from healthy volunteers $(P<0.0001)$. C4d deposition was similar $(1.16 \pm 0.85)$ to that found with serum from healthy volunteers but significantly increased $(P<0.0001)$ relative to aPL negative sera from patients with SLE (Figure 1B). There was no correlation between the magnitude of C1q or C4d deposition (ratio) and aPL levels in patients with SLE (data not shown).

Sera from patients with primary aPL or APS also showed increased C1q and C4d deposition compared with normal serum (Figure 2). However, relative to results obtained with normal serum only increases in C1q deposition were statistically significant (aPL only, $P=0.03$; aPL with APS, $P=0.06$ ). There was a trend towards increased $C 4 \mathrm{~d}$ deposition using sera from patients with aPL only $(P=0.09)$ or aPL with APS $(P=0.10)$, but these did not reach statistical significance.

To further evaluate the relationship between complement fixation, aPL level and thrombosis, patient sera were designated as either 'complement positive' $(\mathrm{C}+)$ or 'complement negative' $(\mathrm{C}$ -). Complement positivity was defined as a ratio greater than 2.0 for serum C1q or C4d deposition relative to control serum. This cutoff represents in-situ C1q or C4d deposition exceeding two standard deviations above the normal reference mean (>95th percentile). As 
shown in Table 3, complement positive sera showed higher IgG APhL and anti- $\beta 2 \mathrm{GP1}$ levels, regardless of the patient population. Notably, only three of 88 aPL negative patients with SLE were complement positive by this definition.

In SLE patients with aPL, complement positivity was significantly associated with a history of arterial thrombosis (Table 4). Reported arterial events were heterogeneous and included predominantly $(68 \%)$ cerebrovascular accidents. No association was noted with history of venous thrombosis, foetal loss or overall incidence of thrombosis in this population. Complement positivity conferred a 2.74 greater odds for arterial thrombosis in SLE patients with aPL $(P=0.03,95 \% \mathrm{CI}=1.08-6.94)$. After adjusting for APhL or anti- $\beta 2 \mathrm{GP} 1$ level in a multivariate analysis, the odds ratio decreased to 2.04 (95\% CI $=0.76-5.50)$ and 2.27 (95\% $\mathrm{CI}=0.86-6.0)$ (not statistically significant), suggesting possible confounding effects of aPL level on complement activation. Interestingly, no association emerged between complement fixation and thrombosis in patients with primary aPL.

To further evaluate the relationship between aPL level, complement fixation and arterial thrombosis in SLE patients, the positive predictive value (PPV) for arterial thrombosis was compared for a positive $\mathrm{APhL}$ level alone and the combination of a positive $\mathrm{APhL}$ level with a positive complement fixation ratio $\geq 2.0$. A positive $\mathrm{APhL}$ level alone had a PPV of $41 \%$ for arterial thrombosis and a negative predictive value (NPV) of $61 \%$. In the presence of a simultaneously positive complement fixation test, the PPV increased to $54 \%$, with a NPV of $68 \%$. If only C4d deposition on heterologous platelets was used as a measure of complement fixation, the number of false positive test results decreased and the PPV increased to $70 \%$ (NPV $69 \%$ ). Moreover, if the positive cutoff for $\mathrm{C} 4 \mathrm{~d}$ deposition was raised to a ratio greater than 2.5, the PPV for arterial thrombosis for the combination of a positive APhL level with a positive complement fixation increased to 83\% (NPV 64\%).

\section{Platelet activation}

Additional studies compared the ability of a subgroup of sera (selected based upon availability), designated complement positive or negative, to activate heterologous platelets in suspension, using a ${ }^{14} \mathrm{C}$ serotonin release assay. The data are summarized in Table 5. Statistically significant increases in ${ }^{14} \mathrm{C}$-serotonin secretion were observed following platelet exposure to complement positive sera, regardless of the patient population.

\section{Discussion}

Results from the present study show that sera from patients with aPL exhibit an enhanced capacity to activate the classical pathway of complement, defined by C1q and/or C4d deposition on heterologous platelets. In aPL patients with underlying SLE, deposition of C1q and especially $\mathrm{C} 4 \mathrm{~d}$, exceeding twice the level observed with normal serum, was associated with an increased incidence of arterial thrombosis. Despite enhanced classical pathway complement activation by sera from patients with primary aPL, a similar association with arterial thrombosis was not observed. A significant association, however, was noted between the presence of aPL IgG and positive complement fixation on heterologous platelets in all patients, although the correlation between the magnitude of aPL levels and the extent of complement deposition was poor, likely reflecting heterogeneity in the complement fixing ability of the autoantibodies present.

At the time of inclusion, none of the patients were receiving any form of heparin therapy. This is relevant because heparin can inhibit complement activation. ${ }^{28}$ However, some patients in both the SLE and APSCORE registries were receiving anticoagulation therapy with warfarin. In the APSCORE cohort, 4/47 aPL patients were receiving warfarin, compared with 59/96 patients with APS. In the SLE cohort, 3/91 aPL negative patients and 28/78 aPL positive 
patients were treated with warfarin. Although vitamin K antagonism is not expected to directly impact the complement system, thrombin generation may indirectly enhance complement activation. ${ }^{23}$ For this reason, in-situ complement activation on immobilized platelets was assayed in the presence of a direct thrombin inhibitor (PPACK).

The association between serum complement fixation and arterial thrombosis in patients with SLE and aPL, but not primary aPL, may suggest that other SLE-related factors are mechanistically important for arterial thrombosis in the setting of aPL-mediated complement activation. Alternatively, or in addition, the variance in C1q and C4d deposition observed with sera from patients with primary aPL was greater than that noted with sera from normal controls or patients with SLE. This may reflect preanalytical variables associated with serum preparation and storage that could affect complement activation 29 and may have contributed to the apparent lack of association between complement positivity and thrombotic events in patients with primary aPL.

The following mechanism for the interaction between aPL, complement activation and arterial thrombosis may be plausible. Circulating aPL auto-antibodies bind to the platelet surface, creating an immune complex that, depending on antibody isotype, is recognized by complement (i.e., C1q). In-situ complement activation subsequently occurs on the platelet surface, as evidenced by $\mathrm{C} 4 \mathrm{~d}$ deposition. Platelet activation by complement, 30 as observed in vitro using the serotonin release assay, and the generation of complement-derived inflammatory mediators 31 may promote arterial thrombosis. In the setting of SLE, vascular injury may create an environment that predisposes to platelet accumulation and subsequent insitu complement activation.

The complement system plays an important role in the pathogenesis of SLE. Complement deficiencies in the classical pathway (C1q, C4 and C2) predispose to the development of autoimmunity by a variety of mechanisms including impaired clearance of immune complexes and apoptotic cells, as well as aberrant tolerance induction and changes in cytokine regulation. 26,27 During SLE flares, the complement system is activated, giving rise to partial deficiency or dysfunction due to consumption. ${ }^{27}$ Complement activation takes part in inflammatory reactions that give rise to tissue and organ damage. ${ }^{26}$ Because the extent of complement consumption in SLE patients likely impacts measurable complement deposition on platelets in the current assay, a limitation of our study is the inability to assay C1q levels and C4 levels in all serum samples due to limited sample volume. Thus, it was not possible to normalize complement deposition to serum complement levels. Indeed, C4d deposition was lowest in samples with lowest $\mathrm{C} 4$ levels. This may have increased false negative results.

Another significant limitation of the present study is the inability to correlate complement activation with lupus anticoagulant information. Determination of lupus anticoagulant status is indeed important for an APS series. Although these data were available for most samples in the APSCORE cohort (Table 2), platelet-poor plasma samples were unavailable for analysis in the SLE cohort, and thus, concurrent lupus anticoagulant measurements could not be made. It should be noted, however, that various APSCORE and patients with SLE were on warfarin therapy, which may interfere with lupus anticoagulant measurement. Thus, even if lupus anticoagulant data were available for all patients, the percentage of true positive patients would be overestimated.

The role of complement in APS is under intense investigation. Multiple pathogenic mechanisms have been suggested. ${ }^{32}$ Exposure of human endothelial cells to aPL has been shown to result in tissue factor production and upregulation of adhesion molecules. ${ }^{33}$ Although the cause of tissue injury in APS is likely to be multifactorial, 32,34 a substantial body of evidence shows that complement activation is a requirement for pregnancy complications and 
thrombosis. ${ }^{12}$ Potentially, aPL-mediated complement fixation contributes to arterial thrombosis via production of inflammatory mediators: $\mathrm{C} 3 \mathrm{a}$ responsible for leukocyte recruitment ${ }^{35}$ and C5a stimulating endothelial cell production of tissue factor. ${ }^{36}$ The presence of receptors for anaphylatoxins has also been described in human coronary plaques, suggesting that they may play a role in plaque formation/progression. ${ }^{37}$

In patients with SLE, cross-sectional and prospective cohort studies show a predictive role of aPL for future vascular events. ${ }^{38,39}$ However, patients with SLE are at significantly increased risk of premature atherosclerosis and/or thrombosis, ${ }^{40,41}$ regardless of aPL status, and a significant aPL profile does not appear to be predictive of thrombosis in individual patients with SLE. ${ }^{42}$ Moreover, a positive aPL level does not predict the location of thrombotic events (arterial, venous, pregnancy complications). Results from the present study suggest that the ability of sera with positive aPL levels to substantially activate complement on platelets may be associated with arterial thrombosis in patients with SLE, particularly when the complement fixation assay is made more stringent by using $\mathrm{C} 4 \mathrm{~d}$ generation as the endpoint. This may suggest that observed C1q deposition on heterologous platelets reflects the presence of complement fixing antibodies on platelets, whereas the in-situ generation of $\mathrm{C} 4 \mathrm{~d}$ is indicative of the activation of the classical complement system and its inflammatory sequelae. The current study did not have sufficient power to distinguish between specific arterial thrombotic events, such as stroke and myocardial infarction. The majority of patients with arterial thrombotic events (22 of 32) had a history of cerebrovascular accidents, and a history of myocardial infarction was reported for two patients. Other reported arterial thrombotic events included digital, splenic and adrenal infarcts.

In summary, the present study provides further insight into potential pathophysiologic mechanisms associated with thrombotic complications in SLE patients with aPL. Prospective studies are warranted to determine whether direct measurement of C1q and $\mathrm{C} 4 \mathrm{~d}$ deposition on circulating platelets, or enhanced complement fixation on heterologous and/or homologous platelets in situ, may be useful in predicting arterial thrombosis risk in patients with SLE. This information could contribute to more targeted therapeutic interventions in patients having SLE with aPL.

\section{Acknowledgements}

This work was supported in part by grants HL67211 (EIBP) and A1060866 (BG) from the National Institutes of Health, the Mary Kirkland Center for Lupus Research (DRA and JES) and American Heart Association Heritage Affiliate postdoctoral award \#0625900T to Wei Yin.

\section{References}

1. Giannakopoulos B, Passam F, Rahgozar S, Krillis SA. Current concepts on the pathogenesis of the antiphospholipid syndrome. Blood 2007;109:422-430. [PubMed: 16985176]

2. Wilson WA, Gharavi AE, Koike T, et al. International consensus statement on preliminary classification criteria for definite antiphospholipid syndrome: report of an international workshop. Arthritis Rheum 2000;42:1309-1311. [PubMed: 10403256]

3. Petri M. Epidemiology of the antiphospholipid antibody syndrome. J Autoimmun 2000;15:145-151. [PubMed: 10968901]

4. Machin SJ. Platelets and antiphospholipid antibodies. Lupus 1996;5:386-387. [PubMed: 8902766]

5. Simantov R, LaSala JM, Lo DK, Gharavi AE, Sammaritano LR, Salmon JE. Activation of cultured vascular endothelial cells by antiphospholipid antibodies. J Clin Invest 1995;96:2211-2219. [PubMed: 7593607]

6. Pierangeli SS, Colden-Stanfield M, Liu X, Barker JH, Anderson GL, Harris EN. Antiphospholipid antibodies from antiphospholipid syndrome patients: activation of endothelial cells in vitro and in vivo. Circulation 1999;99:1007-2002. 
7. DeJong A, Ziboh V, Robbins D. Antiphospholipid antibodies and platelets. Curr Rheumatol Rep 2000;2:238-245. [PubMed: 11123065]

8. Piona A, la Rosa L, Tincani A, et al. Placental thrombosis and fetal loss after passive transfer of mouse lupus monoclonal or human poly-clonal anti-cardiolipin antibodies in pregnant naïve BALB/c mice. Scand J Immunol 1995;41:427-432. [PubMed: 7725061]

9. Shi W, Chong BH, Chesterman CN. $\beta_{2}$-glycoprotein 1 is a requirement for anticardiolipin antibodies binding to activated platelets: differences with lupus anticoagulants. Blood 1993;81:1255-1262. [PubMed: 8443387]

10. Levine JS, Branch W, Rauch J. The antiphospholipid syndrome. N Engl J Med 2002;346:752-763. [PubMed: 11882732]

11. Salmon JE, Girardi G. The role of complement in antiphospholipid syndrome. Curr Dir Autoimmun 2004;7:133-148. [PubMed: 14719378]

12. Pennings MT, Derksen RH, van Lummel M, et al. Platelet adhesion to dimeric beta-glycoprotein 1 under conditions of flow is mediated by at least two receptors: glycoprotein Ib alpha and apolipoprotein E receptor 2'. J Thromb Haemost 2007;5:369-377. [PubMed: 17096706]

13. Davis WD, Brey RL. Antiphospholipid antibodies and complement activation in patients with cerebral ischemia. Clin Exp Immunol 1992;10:445-460.

14. Shinzato MM, Bueno C, Trindade Viana VS, Borba EF, Goncalves CR, Bonfa E. Complement-fixing activity of anticardiolipin antibodies in patients with and without thrombosis. Lupus 2005;14:953958. [PubMed: 16425575]

15. Navratil JS, Manzi S, Kao AH, et al. Platelet C4d is highly specific for systemic lupus erythematosus. Arthritis Rheum 2006;54:670-674. [PubMed: 16447243]

16. Peerschke EIB, Yin W, Grigg SE, Ghebrehiwet B. Blood platelets activate the classical pathway of human complement. J Thromb Haemost 2006;4:2035-2042. [PubMed: 16961611]

17. Tan EM, Cohen AS, Fries JF, et al. The 1982 revised criteria for the classification of systemic lupus erythematosus. Arthritis Rheum 1982;25:1271-1277. [PubMed: 7138600]

18. Alpert D, Mandl LA, Erkan D, Yin W, Peerschke EI, Salmon JE. Anti- heparin platelet factor 4 antibodies in systemic lupus erythematosus are associated with IgM antiphospholipid antibodies and the antiphospholipid syndrome. Ann Rheum Dis 2008;67:395-401. [PubMed: 17644539]

19. Merkel PA, Chang Y, Pierangeli SS, Harris EN, Polisson RP. Comparison between the standard anticardiolipin antibody test and a new phospholipid test in patients with connective tissue diseases. J Rheumatol 1999;26:591-596. [PubMed: 10090168]

20. Pierangeli S, Henderson V, Pappalardo E, et al. Evaluation of several ELISA tests used in the diagnosis of antiphospholipid syndrome in a large cohort of patient samples. Arthritis Rheum 2006;54 (Suppl):S553.

21. Harris EN, Pierangeli S, Birch D. Anticardiolipin wet workshop report. Fifth International Symposium on antiphospholipid antibodies. Am J Clin Pathol 1994;101:616-624. [PubMed: 7909979]

22. Peerschke EI, Zucker MB, Grant RA, Egan JJ, Johnson MM. Correlation between fibrinogen binding to human platelets and platelet aggregability. Blood 1980;55:841-847. [PubMed: 6767512]

23. Huber-Lang M, Sarma SV, Zetoune FS, et al. Generation of C5a in the absence of C3: a new complement pathway. Nat Med 2006;12:682-687. [PubMed: 16715088]

24. Peerschke EI. Induction of human platelet fibrinogen receptor by epinephrine in the absence of released ADP. Blood 1982;60:71-77. [PubMed: 6282365]

25. Frank MM. Complement in the pathophysiology of human disease. N Engl J Med 1987;316:15251530. [PubMed: 3295544]

26. Pickering MC, Botto M, Taylor PR, Lachmann PJ, Walport MJ. Systemic lupus erythematosus, complement deficiency, and apoptosis. Adv Immunol 2000;76:227-324. [PubMed: 11079100]

27. Truedsson L, Bengtsson AA. Sturfelt, G Complement deficiencies and systemic lupus erythematosus. Autoimmunity 2007;40:560-566. [PubMed: 18075790]

28. Tyrell DJ, Kilfeather S, Page CP. Therapeutic uses of heparin beyond its traditional role as an anticoagulant. Trends Pharmacol Sci 1995;16:198-204. [PubMed: 7652929] 
29. Mollnes TE, Garred P, Bergseth G. Effect of time, temperature and anticoagulants on in vitro complement activation: consequences for collection and preservation of samples to be examined for complement activation. Clin Exp Immunol 1988;73:484-488. [PubMed: 2463123]

30. Sims PJ, Rollins SA, Wiedmer T. Regulatory control of complement on blood platelets. Modulation of platelet procoagulant reponses by a membrane inhibitor of the C5b-9 complex. J Biol Chem 1989;264:19228-19235. [PubMed: 2808422]

31. Markiewski MM, Lambris JD. The role of complement in inflammatory diseases from behind the scenes into the spotlight. Am J Pathol 2007;171:715-727. [PubMed: 17640961]

32. Mackworth-Young CG. Antiphospholipid syndrome: multiple mechanisms. Clin Exp Immunol 2004;136:393-401. [PubMed: 15147339]

33. Kornberg A, Blank M, Kaufman S, Shoenfeld Y. Induction of tissue factor-like activity in monocytes by anti-cardiolipin antibodies. J Immunol 1994;153:1328-1332. [PubMed: 8027560]

34. Meroni PL, Borghi MO, Raschi E, et al. Inflammatory response and the endothelium. Thromb Res 2004;114:329-334. [PubMed: 15507262]

35. Jagels MA, Daffern PJ, Hugli TE. C3a and C5a enhance granulocyte adhesion to endothelial and epithelial cell monolayers: epithelial and endothelial priming is required for C3a-induced eosinophil adhesion. Immunopharmacology 2000;46:209-222. [PubMed: 10741901]

36. Ikeda K, Nagasawa K, Horiuchi T, Tsuru T, Nishizaka H, Niho Y. C5a induces tissue factor activity on endothelial cells. Thromb Haemost 1997;77:294-398. [PubMed: 9157584]

37. Oksjoki R, Laine P, Helske S, et al. Receptors for the anaphylatoxins C3a and C5a are expressed in human atherosclerotic coronary plaques. Atherosclerosis 2007;195:90-99. [PubMed: 17234193]

38. Petri, M. Classification and epidemiology of the antiphospholipid syndrome. In: Asherson, RA.; Cervera, R.; Piette, JC.; Shoenfeld, Y., editors. The antiphospholipid syndrome II: autoimmune thrombosis. Amsterdam: Elsevier; 2002. p. 11-20.

39. Rand JH. Molecular pathogenesis of the antiphospholipid syndrome. Circ Res 2002;90:29-37. [PubMed: 11786515]

40. Mok CC. Accelerated atherosoclerosis, arterial thromboembolism, and preventive strategies in systemic lupus erythematosus. Scand J Rheumatol 2006;35:85-95. [PubMed: 16641040]

41. Haque S, Bruce IN. Therapy insight: systemic lupus erythematosus as a risk factor for cardiovascular disease. Nat Clin Pract Cardiovasc Med 2005;2:423-430. [PubMed: 16119705]

42. Erkan D. Lupus and Thrombosis. J Rheumatol 2006;33:1715-1717. [PubMed: 16960931] 

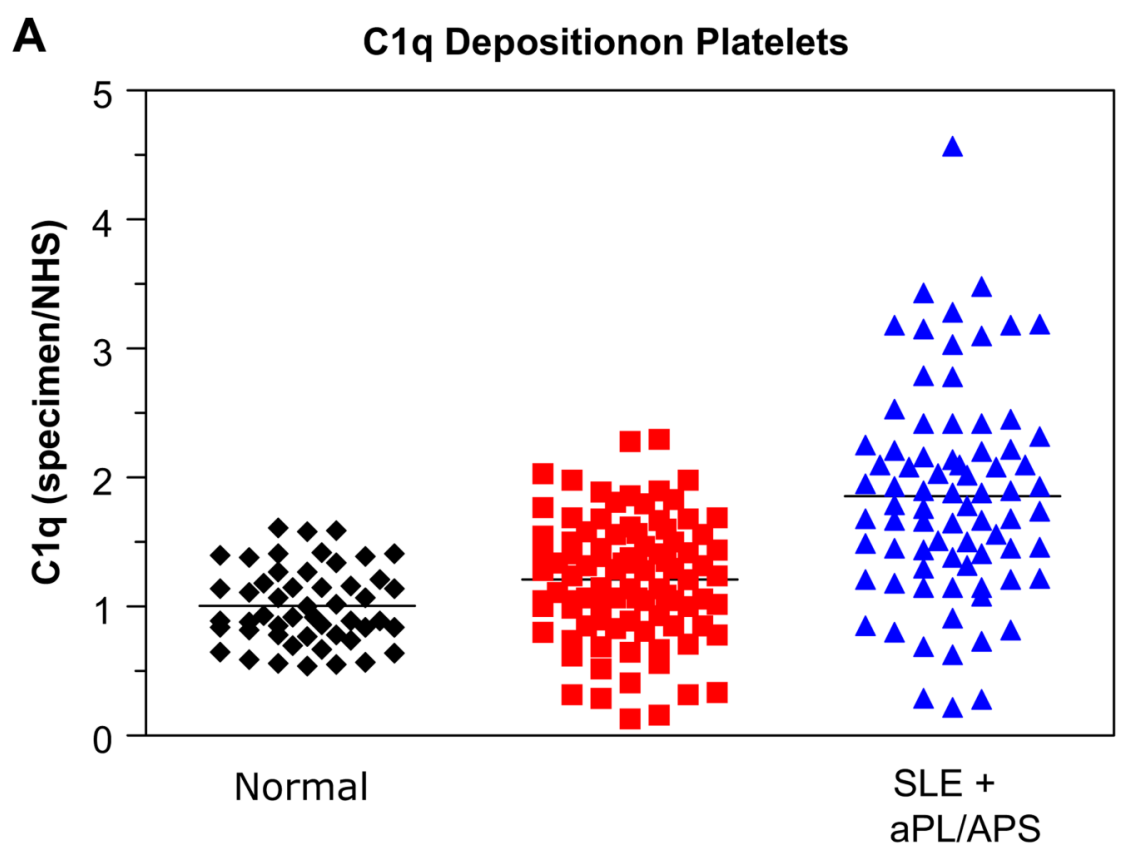

B

C4d Depositionon Platelets

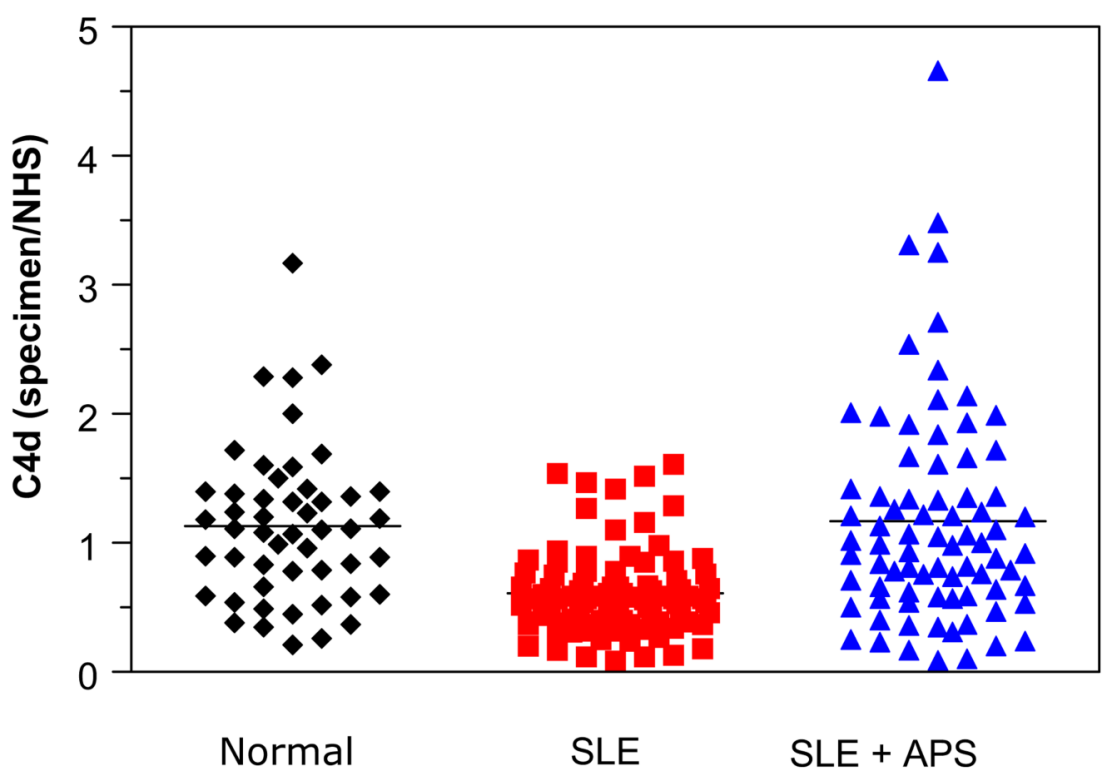

Figure 1.

Complement fixation on heterologous platelets by serum from healthy volunteers (normal, $n$ $=50)$, patients with SLE without aPL $(n=91)$ and patients with SLE and APL or APS $(n=78)$. Classical pathway C1q (Panel A) and C4d (Panel B) deposition are shown. Each data point represents results for a single patient. The horizontal line indicates mean complement component deposition for the group. Complement deposition is expressed as a ratio relative to a normal human serum pool (NHS), used as an internal assay standard, as described in Methods. 
A

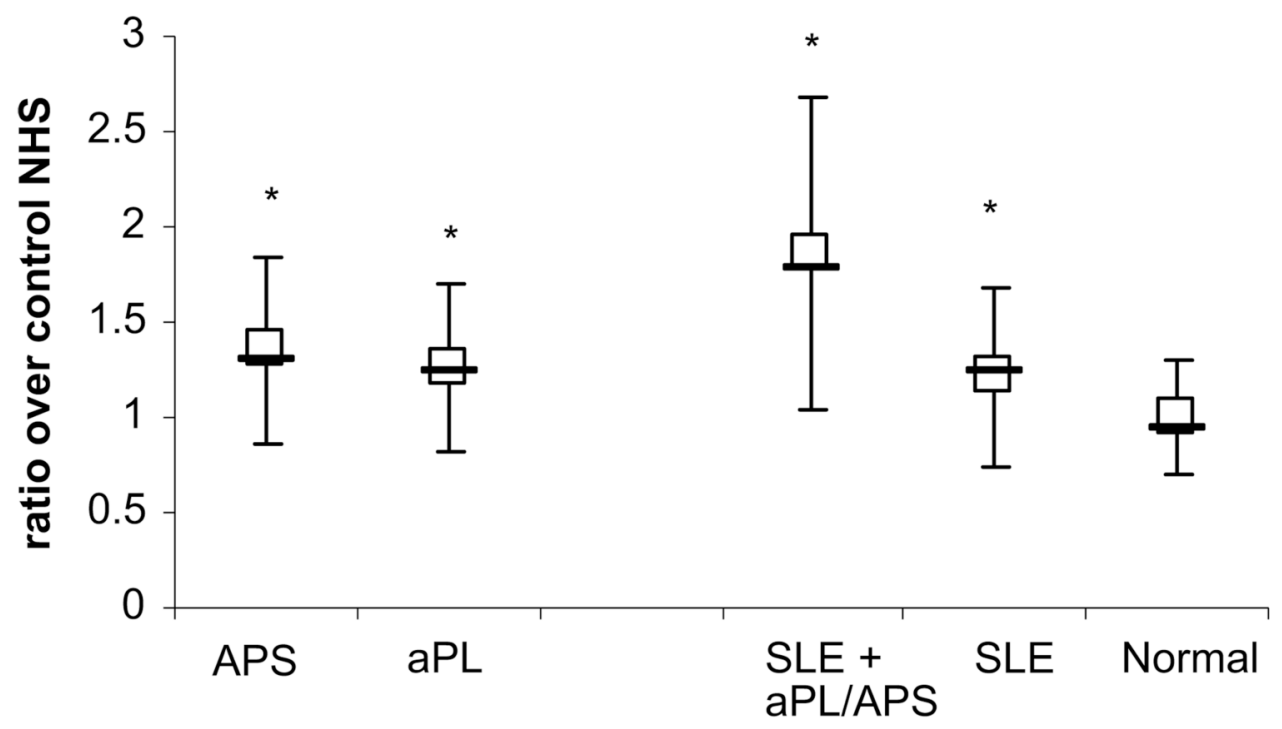

B

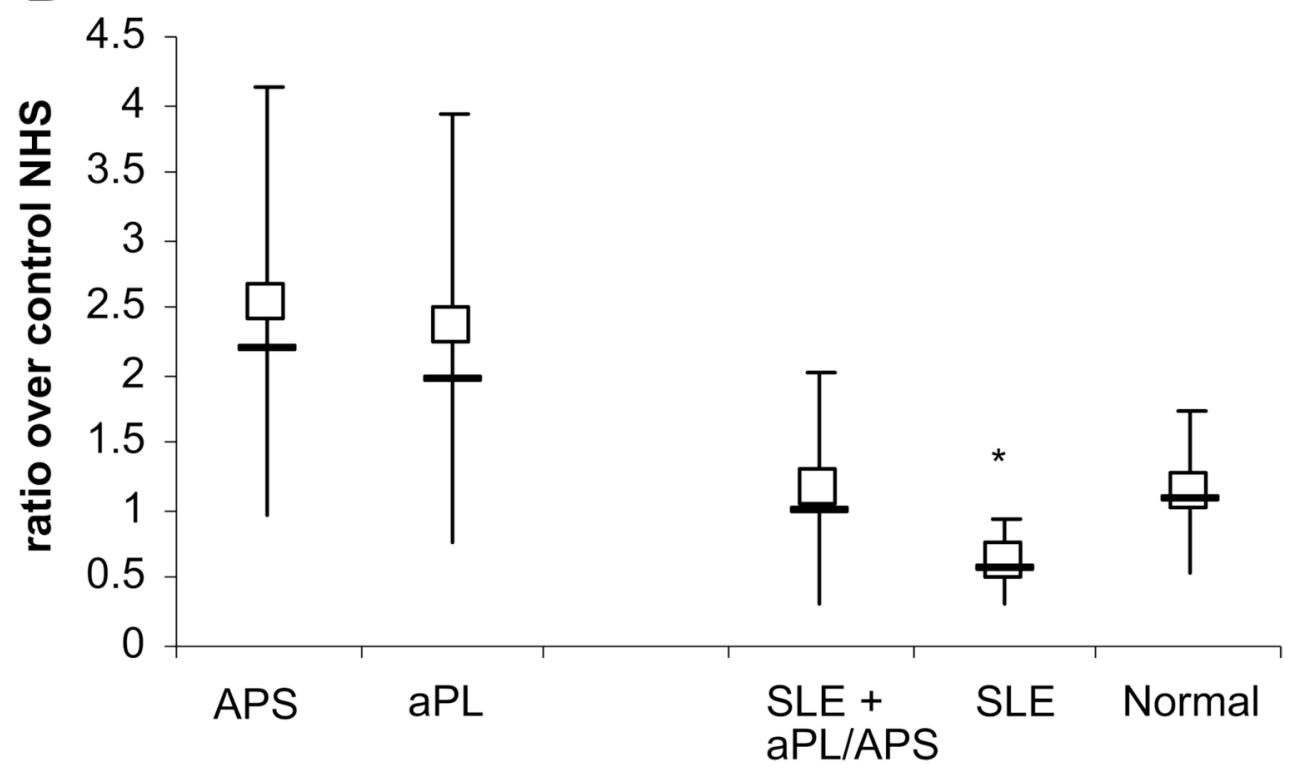

Figure 2.

Comparison of complement fixation on heterologous platelets by serum samples from patients with primary aPL, SLE with aPL. The data depict mean (horizontal line), median (box) and standard deviation for C1q (Panel A) and C4d (Panel B) deposition for serum samples from patients with primary aPL with APS (APS, $n=96$ ), primary aPL only (aPL, $n=57$ ), SLE with aPL or APS (SLE +aPL/APS, $n=78$ ) and SLE without aPL (SLE, $n=91$ ). Complement fixation by serum from healthy volunteers $(n=50$, normal) is shown for reference. Complement deposition is expressed as a ratio relative to an internal normal human serum standard, as described in Methods. The asterix (*) denotes statistically significant differences in complement deposition compared with normal $(P<0.05)$. 
Peerschke et al.

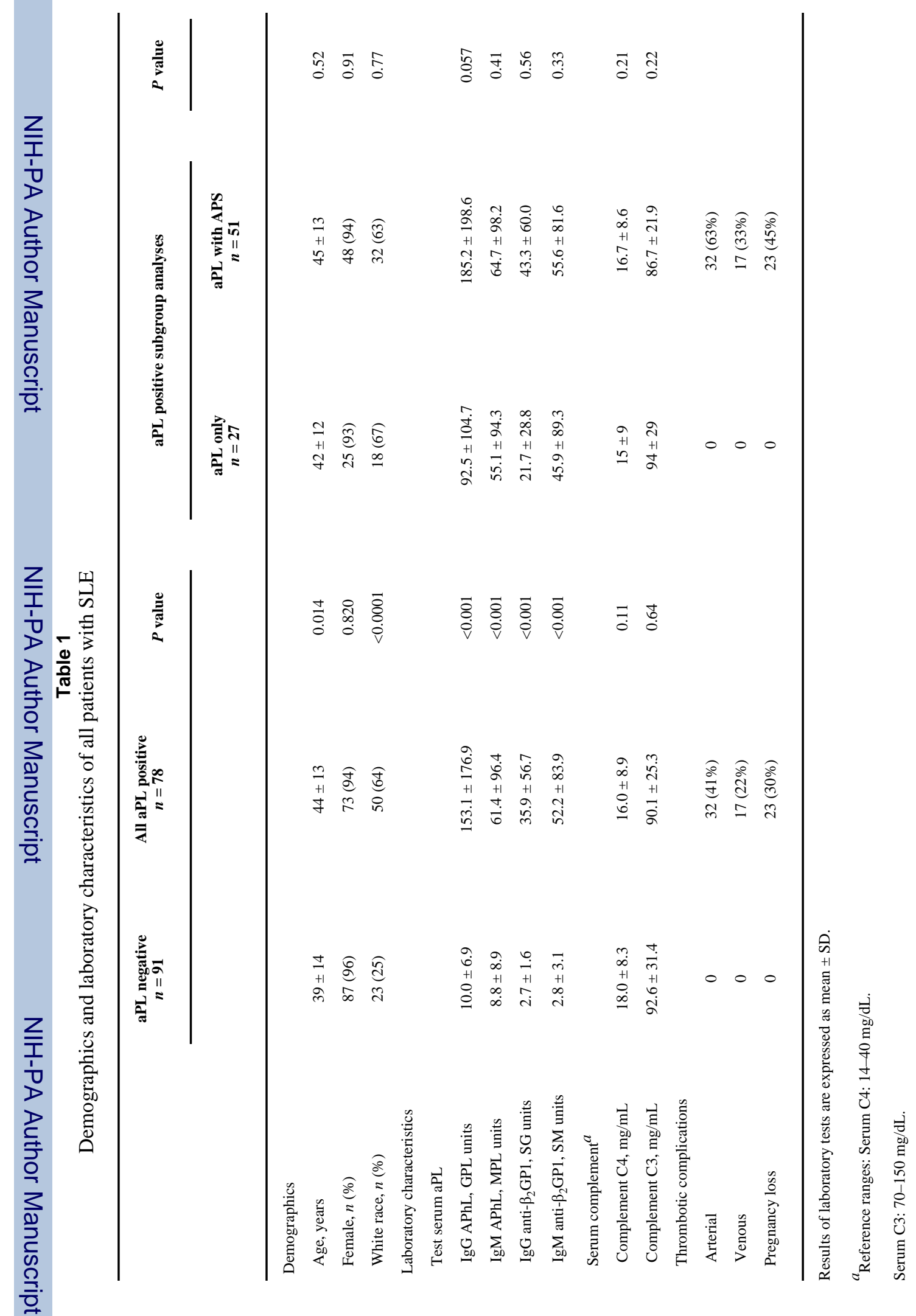


Table 2

Demographics and laboratory characteristics of all patients with primary aPL

\begin{tabular}{|c|c|c|c|}
\hline & $\begin{array}{c}\text { aPL without APS } \\
\qquad n=57\end{array}$ & $\begin{array}{c}\text { aPL with APS } \\
n=96\end{array}$ & $P$ value \\
\hline \multicolumn{4}{|l|}{ Demographics } \\
\hline Age, years & $40 \pm 13$ & $40 \pm 11$ & 0.535 \\
\hline Female, $n(\%)$ & $60(88 \%)$ & $68(80 \%)$ & 0.213 \\
\hline White race, $n(\%)$ & $58(85 \%)$ & $64(75 \%)$ & 0.197 \\
\hline \multicolumn{4}{|c|}{ Laboratory characteristics } \\
\hline \multicolumn{4}{|l|}{ Anticardiolipin } \\
\hline IgG, GPL units & $57 \pm 62$ & $84 \pm 77$ & 0.017 \\
\hline IgM, MPL units & $38 \pm 69$ & $21 \pm 39$ & 0.120 \\
\hline \multicolumn{4}{|l|}{ Anti- $\beta_{2}$ GP1 } \\
\hline IgG, SG units & $47 \pm 67$ & $84 \pm 126$ & 0.006 \\
\hline IgM, SM units & $53 \pm 74$ & $47 \pm 109$ & 0.472 \\
\hline \multicolumn{4}{|l|}{ Lupus anticoagulant } \\
\hline Positive, $n+\ln (\%)$ & $32 / 49(65 \%)$ & $71 / 84(85 \%)$ & 0.272 \\
\hline \multicolumn{4}{|c|}{ Thrombotic complications } \\
\hline Arterial, $n(\%)$ & 0 & $44(46 \%)$ & \\
\hline Venous & 0 & $036(38 \%)$ & \\
\hline Pregnancy & 0 & $037(39 \%)$ & \\
\hline
\end{tabular}

Values are presented as mean $\pm \mathrm{SD}$.

$n+/ n$, number of patients with finding/number of patients with available data. 
Peerschke et al.

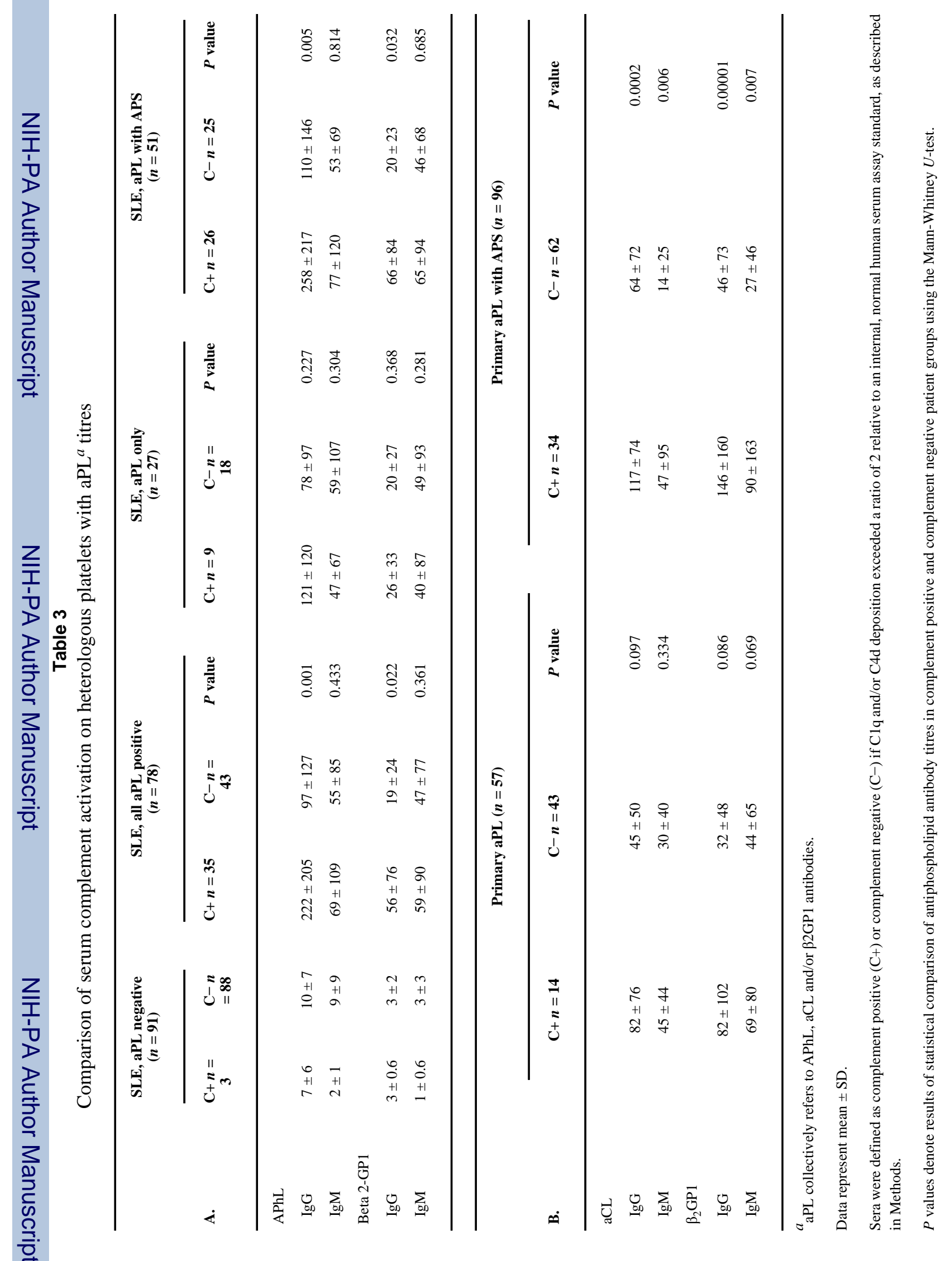

Lupus. Author manuscript; available in PMC 2009 July 9. 
Peerschke et al.

Page 16

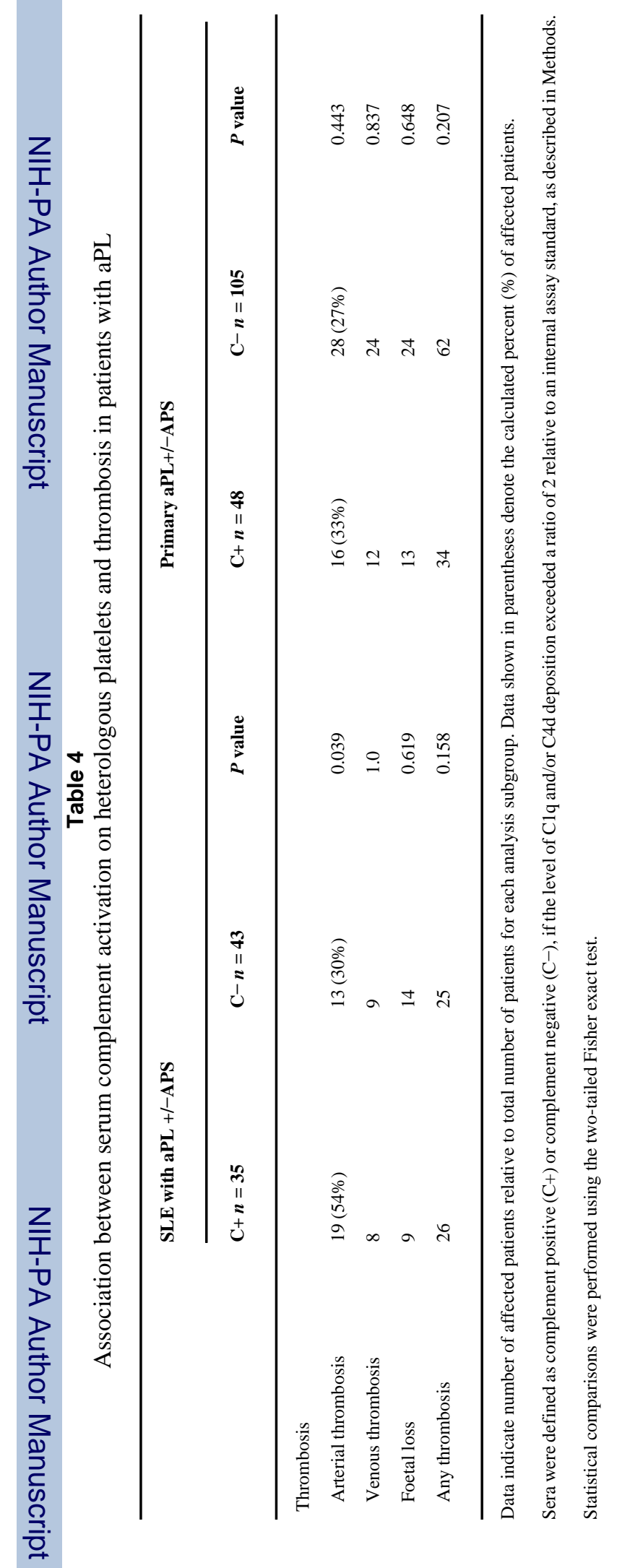

Lupus. Author manuscript; available in PMC 2009 July 9. 
Table 5

Comparison of platelet activation by complement positive or complement negative sera from patients with aPL

\begin{tabular}{llll}
\hline${ }^{14}$ C-Serotonin release (\%) & & & P value \\
\hline Patients & C+ & C- & 0.002 \\
\hline SLE with aPL & $55 \pm 11(n=12)$ & $28 \pm 15(n=5)$ & 0.004 \\
Primary aPL & $70 \pm 10(n=7)$ & $44 \pm 10(n=4)$ & \\
Normal & NA & $14 \pm 8(n=5)$ & \\
\hline
\end{tabular}

Platelet activation was assessed by ${ }^{14} \mathrm{C}$-serotonin release assay. Results are expressed as mean $\pm \mathrm{SD}$.

Patient sera were selected based on sample availability.

Sera were defined as complement positive (C+)if the levels of $\mathrm{C} 1 \mathrm{q}$ and/or $\mathrm{C} 4 \mathrm{~d}$ deposition exceeded twice that obtained with an internal assay standard, as described in Methods. 